\title{
Particle-Based Modeling of Asymmetric Flexible Fibers in Viscous Flows
}

\author{
Xiufeng Yang ${ }^{1,2}$ and Moubin $\mathrm{Liu}^{3,4, *}$ \\ ${ }^{1}$ Institute of Mechanics, Chinese Academy of Sciences, Beijing 100190, China. \\ 2 Department of Mechanical Engineering, Iowa State University, Ames, IA 50011, \\ USA. \\ ${ }^{3}$ BIC-ESAT, College of Engineering, Peking University, Beijing 100187, China. \\ 4 State Key Laboratory for Turbulence and Complex Systems, Peking University, \\ Beijing 100871, China.
}

Received 15 November 2016; Accepted (in revised version) 15 February 2017

\begin{abstract}
The present paper follows our previous work [Yang et al., Phys. Rev. E, 90 (2014), 063011] in which the bending modes of a symmetric flexible fiber in viscous flows were studied by using a coupling approach of smoothed particle hydrodynamics $(\mathrm{SPH})$ and element bending group (EBG). It was shown that a symmetric flexible fiber can undergo four different bending modes including stable U-shape, slight swing, violent flapping and stable closure modes. For an asymmetric flexible fiber, the bending modes can be different. This paper numerically studies the fiber shape, flow field and fluid drag of an asymmetric flexible fiber immersed in a viscous fluid flow by using the SPH-EBG coupling method. An asymmetric number is defined to describe the asymmetry of a flexible fiber. The effects of the asymmetric number on the fiber shape, flow field and fluid drag are investigated.
\end{abstract}

AMS subject classifications: 76M28, 76D99, 74S99

Key words: Fluid-structure interaction, asymmetric flexible fiber, drag force, smoothed particle hydrodynamics (SPH), element bending group (EBG).

\section{Introduction}

Trees, grasses and some animals have to withstand fluid forces such as aerodynamic forces in air or hydrodynamic forces in water. They can change their shapes or postures to adapt the fluid forces. This strategy can change the fluid forces imposed on them. For example, a fish can get forward thrust and use environmental vortices by waving

${ }^{*}$ Corresponding author. Email addresses: xyang@iastate.edu (X. F. Yang), mbliu@pku.edu.cn (M. B. Liu) 
motion [1,2] and a plant can reduce fluid drag by reconfiguration [3,4]. For flexible structures such as most plants, some animals and flags, their shapes become a function of the relative speed between the corresponding object and the ambient fluid. Thus dynamics and flow patterns of the interactions of flexible structures and fluids are much more complex than that of rigid structures and fluids $[5,6]$.

There have been a number of experimental, theoretical and numerical studies on the dynamics of flexible structures interacting with fluid flows over the past decades. Vogel $[3,7]$ experimentally studied the fluid drag and reconfiguration of broad leaves in high winds. The experiments showed that some broad leaves rolled up into cone shapes in strong winds and the drag on the leaves increases slower than the square of the wind speed. Laura et al. [8] also studied the reconfiguration of broad leaves experimentally and numerically. Gossellin et al. [9] investigated experimentally the drag reduction of two flexible plates of different shapes in air. Alben et al. [10,11] experimentally and numerically studied the drag reduction of a flexible fiber immersed in a soap film. Yang et al. $[12,13]$ numerically investigated the bending modes and drag reduction of a flexible fiber immersed in a viscous flow using a coupling method of smoothed particle hydrodynamics (SPH) and element bending group (EBG).

Although a flexible fiber is a very simple structure, the dynamics of a flexible fiber interacting with a viscous flow have not yet been thoroughly explored. Most previous studies on drag reduction of flexible fibers in flows were focused on cases in which a symmetric flexible fiber (fixed on its midpoint) is immersed in a viscous flow. However, there are more frequent cases with asymmetric flexible fibers (fixed on points different from its midpoint) interacting with viscous fluids and these can produce quite different behaviors from symmetric fibers.

Due to the existence of moving interfaces and deformable boundaries, it is usually a big challenge to model fluid-flexible fiber interaction, especially for cases with high Reynolds numbers. As such, a numerical approach which can well treat moving interfaces and deformable boundaries would be appealing in modeling fluid-flexible fiber interaction. Yang et al. $[12,14]$ developed a SPH-EBG coupling method to model the interaction of a flexible fiber interacting with viscous fluids. The SPH method is used to model fluid motion, while the EBG method is used to model fiber motion. Since both SPH [15] and EBG [16] are Lagrangian particle methods, the coupling of these two methods does not lead to significant difficulties. The SPH-EBG coupling method was first used to model red blood cell (RBC) deformation in a shear flow [17]. Later it was extended to model a flexible fiber in a viscous flow $[12,13]$ and dam-break flows impacting on flexible structures [14].

In this paper, the SPH-EBG coupling method is further used to study the dynamics of an asymmetric flexible fiber interacting with a viscous flow including the fiber shape (bending modes), flow field, and drag force. Comparing to the simulation of symmetric cases, there is no more numerical difficulty in simulating asymmetric cases. The numerical method used for this work is nearly the same as the method used in [12]. In order to make the flow field smoother and the method more stable, the artificial viscosity pro- 
posed by Monaghan [18] is used in this work. The details of the numerical method are given in Section 2. The computational settings are given in Section 3. Numerical results and discussions are given in Section 4. Finally, the conclusions are given in Section 5.

\section{Numerical methodology}

\subsection{SPH method}

SPH is a Lagrangian meshfree particle method, which was first proposed to model astrophysical problems $[19,20]$. In recent years, the SPH method was widely used to solve many fluid flow problems such as free surface flows [21-23], viscous incompressible flows [24, 25], and fluid-structure interactions [26, 27]. In the present study, the SPH method is applied to simulate fluid flow. The Lagrangian form of the Navier-Stokes $(\mathrm{N}-\mathrm{S})$ equations are used for viscous fluid

$$
\begin{aligned}
& \frac{d \rho}{d t}=-\rho \nabla \cdot \mathbf{u}, \\
& \frac{d \mathbf{u}}{d t}=\mathbf{g}-\frac{1}{\rho} \nabla p+\frac{\mu}{\rho} \nabla^{2} \mathbf{u},
\end{aligned}
$$

where $\rho$ is the fluid density, $\mathbf{u}$ is the fluid velocity, $\mathbf{g}$ is the body force acting on the fluid, $p$ is the fluid pressure, and $\mu$ is the dynamic viscosity of the fluid.

In $\mathrm{SPH}$, the N-S equations can be written in the following form:

$$
\begin{aligned}
& \frac{d \rho_{a}}{d t}=\sum_{b} m_{b}\left(\mathbf{u}_{a}-\mathbf{u}_{b}\right) \cdot \nabla_{a} W_{a b}, \\
& \frac{d \mathbf{u}_{a}}{d t}=\mathbf{g}_{a}-\sum_{b} m_{b}\left(\frac{p_{a}}{\rho_{a}^{2}}+\frac{p_{b}}{\rho_{b}^{2}}+\Pi_{a b}\right) \nabla_{a} W_{a b}+\sum_{b} \frac{m_{b}\left(\mu_{a}+\mu_{b}\right)\left(\mathbf{r}_{a}-\mathbf{r}_{b}\right) \cdot \nabla_{a} W_{a b}}{\rho_{a} \rho_{b}\left(r_{a b}^{2}+\eta\right)}\left(\mathbf{u}_{a}-\mathbf{u}_{b}\right),
\end{aligned}
$$

where the subscripts $a$ and $b$ denote SPH particles, $m$ is the mass of a SPH particle. $W$ is a kernel function.

$$
W_{a b} \equiv W\left(\mathbf{r}_{a}-\mathbf{r}_{b}, h\right), \quad \nabla_{a} W_{a b} \equiv \frac{d W_{a b}}{d \mathbf{r}_{a b}} \frac{\mathbf{r}_{a b}}{r_{a b}},
$$

here $h$ is a smoothing length used to control the width of the kernel. $\Pi$ is an artificial viscosity proposed by Monaghan [18]

$$
\Pi_{a b}= \begin{cases}\frac{-\alpha c h \mathbf{u}_{a b} \cdot \mathbf{r}_{a b}}{\bar{\rho}_{a b}\left(r_{a b}^{2}+\eta\right)}, & \mathbf{u}_{a b} \cdot \mathbf{r}_{a b}<0, \\ 0, & \mathbf{u}_{a b} \cdot \mathbf{r}_{a b} \geq 0,\end{cases}
$$

where $\bar{\rho}_{a b}=\left(\rho_{a}+\rho_{b}\right) / 2 . \alpha$ is a parameter for controlling the strength of the artificial viscosity. 
Eqs. (2.2a) and (2.2b) are closed by using the following equation of state [24]:

$$
p=c^{2}\left(\rho-\rho_{0}\right) .
$$

Here, $c$ is a numerical speed of sound, $\rho_{0}$ is a reference density of the fluid.

\subsection{EBG method}

The movement and deformation of a flexible fiber is modeled by using the EBG model, which replaces the fiber with particles. The fiber particles can interact with neighboring fiber particles and fluid particles. In the EBG model, the bending moment is transformed to pairs of forces acting on particles $[16,17]$. An EBG is made of two adjacent line segments connecting three neighboring particles (see Fig. 1).

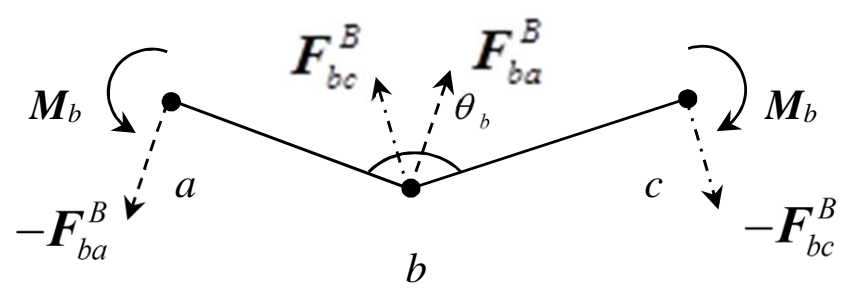

Figure 1: An EBG element is made of two adjacent line segments connecting three neighboring particles. In the EBG model, the bending moment is converted to pairs of forces acting on particles.

According to Newton's second law of motion, the equation for a flexible fiber particle can be written as follows

$$
m \frac{d \mathbf{u}}{d t}=\mathbf{T}+\mathbf{F}^{B}+\mathbf{F}^{D}+\mathbf{g}
$$

where $\mathbf{T}$ denotes the tension acting on a fiber particle from adjacent fiber particles, $\mathbf{F}^{B}$ denotes the force due to EBG bending moment, $\mathbf{F}^{D}$ denotes the fluid force from neighboring fluid (SPH) particles.

The tension acting on particle $b$ from its adjacent particle $a$ is

$$
\mathbf{T}_{b a}=E A\left(\frac{r_{a b}}{r_{a b}^{0}}-1\right) \frac{\mathbf{r}_{a b}}{r_{a b}},
$$

where $E A$ is the tensile rigidity of the flexible fiber, $r_{a b}^{0}$ is the reference distance between particles $a$ and $b$.

The bending force $\mathbf{F}_{b a}^{B}$ acting on particle $b$ from particle $a$ is defined as

$$
\mathbf{F}_{b a}^{B}=\frac{\mathbf{M}_{b} \times \mathbf{r}_{a b}}{r_{a b}^{2}}
$$


where $\mathbf{M}_{b}$ denotes the bending moment acting on particle $b$ (see Fig. 1), which is defined as

$$
M_{b}=\frac{E I\left(\theta_{b}-\theta_{b}^{0}\right)}{r_{b a}+r_{b c}}
$$

where $E I$ and $\theta$ is the bending rigidity and the deflection of the flexible fiber, respectively. $\theta_{b}^{0}$ is the reference deflection at particle $b$.

\subsection{SPH and EBG coupling}

For simulating a flexible fiber in a fluid flow, the SPH particles are used to model fluid and the EBG particles are used to model flexible fiber. The interaction of the fluid and the flexible fiber is simulated by the interaction of neighboring fluid (i.e., SPH) and fiber (i.e., EBG) particles. As fluid and fiber particles are regarded as neighboring particles, it is natural to include fiber particles when calculating forces acting on fluid particles. In other words, for a fluid particle with both neighboring fluid and fiber particles, the total number of particles in summation consists of the total number of neighboring fluid particles and the total number of fiber particles. Therefore, the fiber particles can be regarded as a special type of SPH particles for boundary (or interface) treatment. On one hand, they can interact with regular SPH particles for fluids to render fluid-structure interaction. On the other hand, they can interact with each other as EBG particles. More details can be found in [12].

\section{Computational settings}

The computational settings for the interaction of fluid and flexible fiber is shown in Fig. 2. The fiber is immersed in fluid. At the initial state, the fiber is a straight line in the direction perpendicular to the channel. A point of the fiber is fixed in the midline of the channel. The fixed point cannot move and rotate. The two ends of the fiber are free to move. In order to study the dynamics of an asymmetric fiber, the fixed point of the fiber is not the midpoint of the fiber in most cases. The system is initially at rest. The fluid is driven by

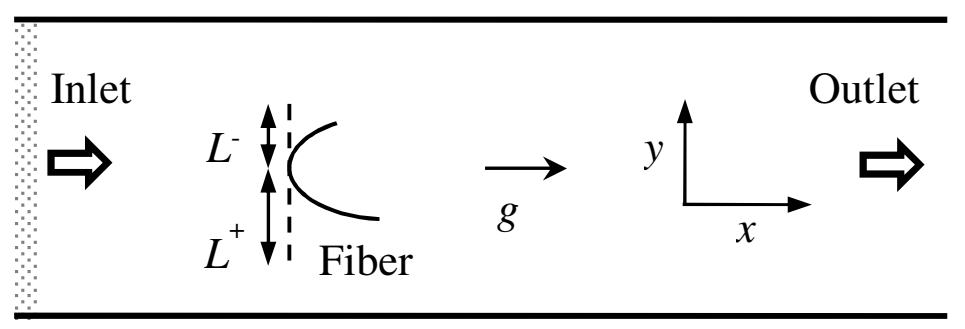

Figure 2: A sketch of the computational settings for fluid-flexible fiber interaction. The dashed line is the initial position of the fiber, while the solid line is the illustration of a bended position of the fiber in the flow. 
Table 1: The length of fibers.

\begin{tabular}{||c|c|c|c|c|c|c|c|c|c||}
\hline Case No. & 1 & 2 & 3 & 4 & 5 & 6 & 7 & 8 & 9 \\
\hline$L(\mathrm{~cm})$ & 3.0 & 3.0 & 3.0 & 3.0 & 4.0 & 4.0 & 4.0 & 4.0 & 4.0 \\
$L^{-}(\mathrm{cm})$ & 1.5 & 1.0 & 0.5 & 0 & 2.0 & 1.5 & 1.0 & 0.5 & 0 \\
$L^{+}(\mathrm{cm})$ & 1.5 & 2.0 & 2.5 & 3.0 & 2.0 & 2.5 & 3.0 & 3.5 & 4.0 \\
$e$ & 0 & $1 / 3$ & $2 / 3$ & 1 & 0 & $1 / 4$ & $1 / 2$ & $3 / 4$ & 1 \\
\hline
\end{tabular}

a body force $g$. In order to let the flow speed increase slowly and linearly, the body force is variable over time. Periodic boundary condition is used in the flow direction. In order to remove the influence of the outlet flow on the inlet flow, a layer of porous media is set in the inlet. In our simulations, the porous media is described by a drag term in the governing equation $[12,27]$.

The channel is $0.4 \mathrm{~m}$ in length and $0.09 \mathrm{~m}$ in width. The density and dynamic viscosity of the fluid are $1000 \mathrm{~kg} / \mathrm{m}^{3}$ and $0.004 \mathrm{Ns} / \mathrm{m}^{2}$, respectively. The bending rigidity of the fiber is $0.002 \mathrm{Jm}$. The length of the fiber is $3 \mathrm{~cm}$ and $4 \mathrm{~cm}$. The position of the fixed point is from the midpoint to the upper end of the fiber. The details of the fiber lengths are shown in Table 1.

In order to describe the asymmetry of the fiber, a non-dimensional parameter, asymmetric number, is defined as

$$
e=\frac{L^{+}-L^{-}}{L}
$$

where $L=L^{+}+L^{-}$is the total length of the fiber, $L^{+}$is the fiber length from the fixed point to the longer end, and $L^{-}$is the fiber length from the fixed point to the shorter end (see Fig. 2). In this paper, the longer end is also the lower end in all the asymmetric cases. According to definition equation (3.1), the asymmetric number $e$ is in the range of 0 to 1 : $e=0$ is the symmetric case, while $e=1$ is the most asymmetric case.

\section{Results and discussions}

\subsection{Fiber shape}

Figs. 3-5 show the fiber shapes with the same fiber length $L=3 \mathrm{~cm}$ but different asymmetric numbers $e=0,1 / 3,2 / 3$ and 1 . In general, for the symmetric case $(e=0)$, the fiber bends symmetrically, which means the two ends of the fiber bends nearly the same; for the asymmetric cases ( $e=1 / 3,2 / 3$ and 1$)$, the fibers bend asymmetrically, which means the lower parts (longer ends) of the fibers bend more than the upper parts (shorter ends). It is obvious that at the same flow velocity, a longer end undergoes a larger fluid force than a shorter end does, thus a longer end is easier to bend in the same flow. It is also shown in Figs. 3-5 that as the asymmetric number increases, the fiber shape becomes more complex. When the asymmetric number $e=1$, the fiber even bends like a flag at high flow velocities. 


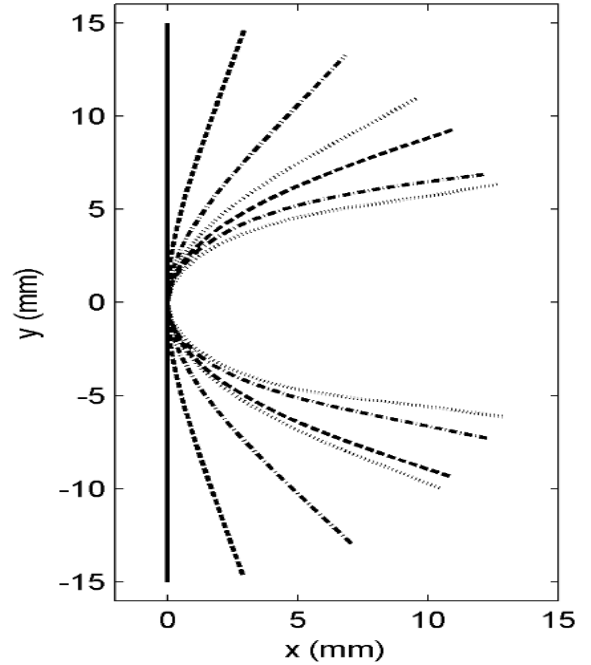

(a)

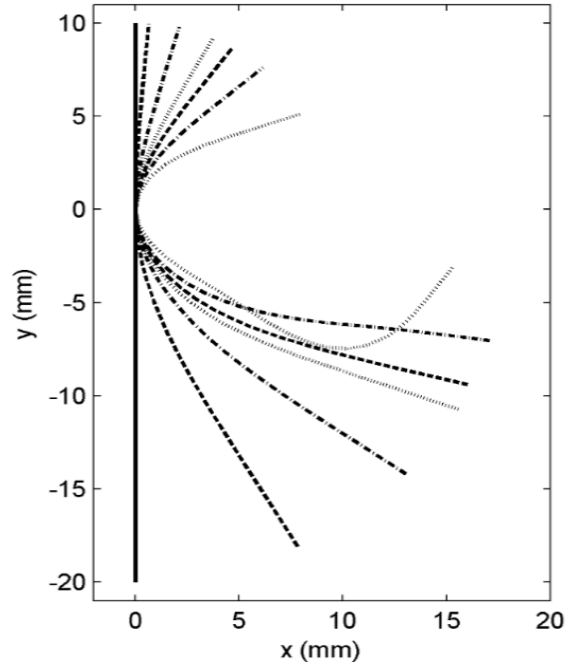

(b)

Figure 3: Comparison of shapes of symmetric $((\mathrm{a}) e=0)$ and asymmetric ((b) $e=1 / 3)$ fibers with the same fiber length $L=3 \mathrm{~cm}$.

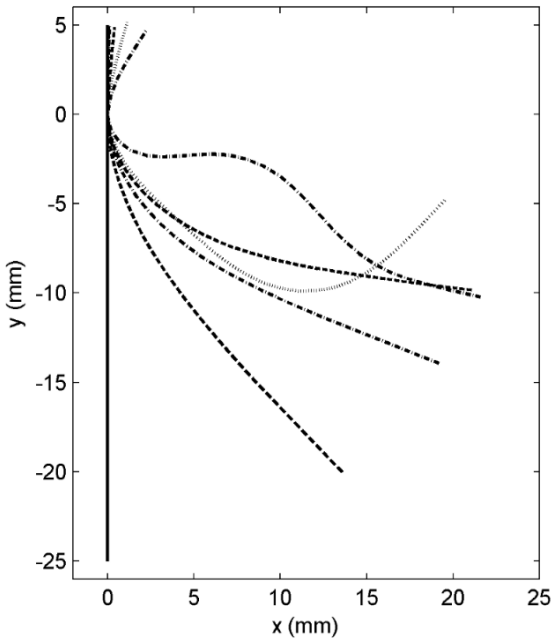

Figure 4: Fiber shapes with fiber length $L=3 \mathrm{~cm}$ and $e=2 / 3$.

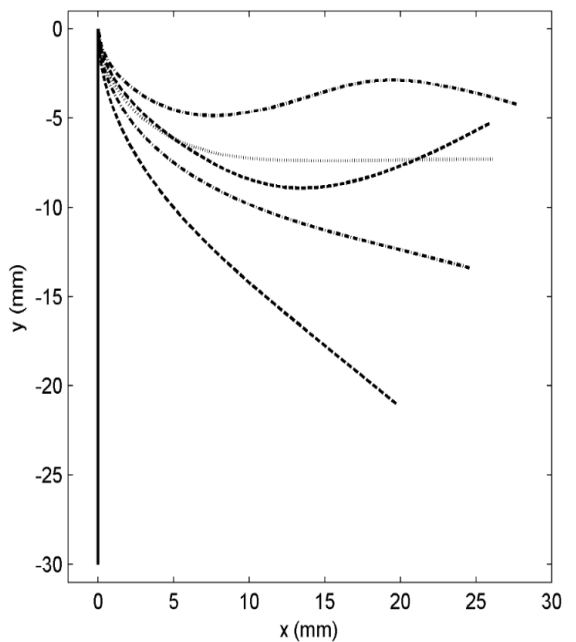

Figure 5: Fiber shapes with fiber length $L=3 \mathrm{~cm}$ and $e=1$.

\subsection{Flow field}

Figs. 6 and 7 show the streamlines of the flow field around the flexible fibers of the same length $L=3 \mathrm{~cm}$ but different asymmetric numbers. It is shown in Figs. 6 and 7 that the flow is symmetric around the symmetric fiber when the flow velocity is $0.5 \mathrm{~m} / \mathrm{s}$, while 

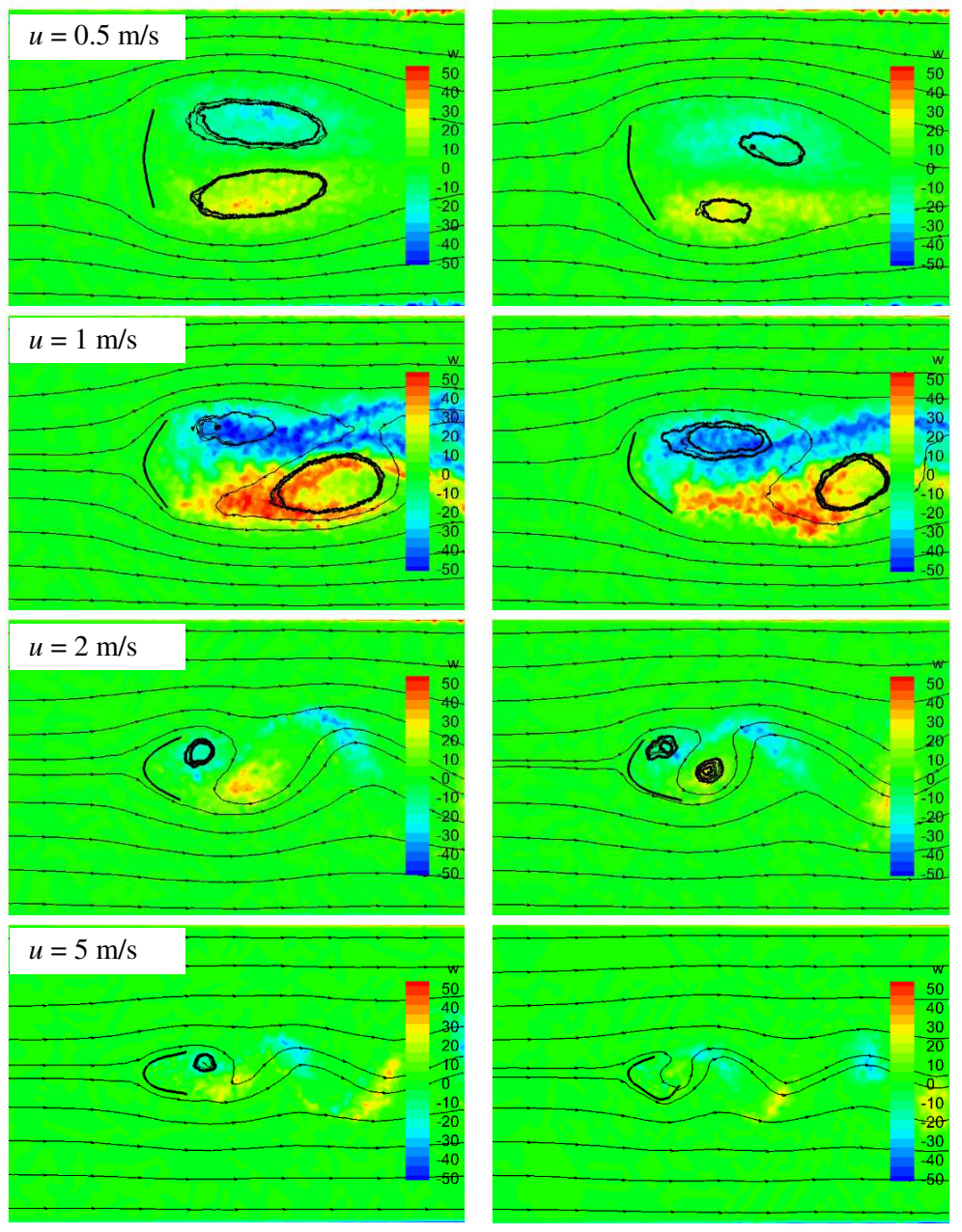

Figure 6: The streamlines for the flexible fiber of length $L=3 \mathrm{~cm}$ but different asymmetric number $e=0$ (left) and $e=1 / 3$ (right) at different flow velocities. The color shows the angular velocity of SPH particles.

the flow fields are asymmetric around the asymmetric fibers. However, as flow velocity increases, the so-called Karman vortex streets appear behind all the fibers regardless of their asymmetric numbers. Because of vortex shedding effects, all the fibers in the four cases shown in Figs. 6 and 7 begin to flap. As the asymmetric number increases, the longer end of the fiber flaps more and forms a more complex shape. We can see from Figs. 6 and 7 that at flow velocity of $5 \mathrm{~m} / \mathrm{s}$, the symmetric fiber $(e=0)$ only bends around the fixed point, however, besides the fixed points, the asymmetric fibers $(e>0)$ also bend around their free longer ends: for the fiber of $e=1 / 3$, there is one more bend on the free longer end; for the fibers of $e=2 / 3$ and 1 , there are two more bends on the free longer ends. 

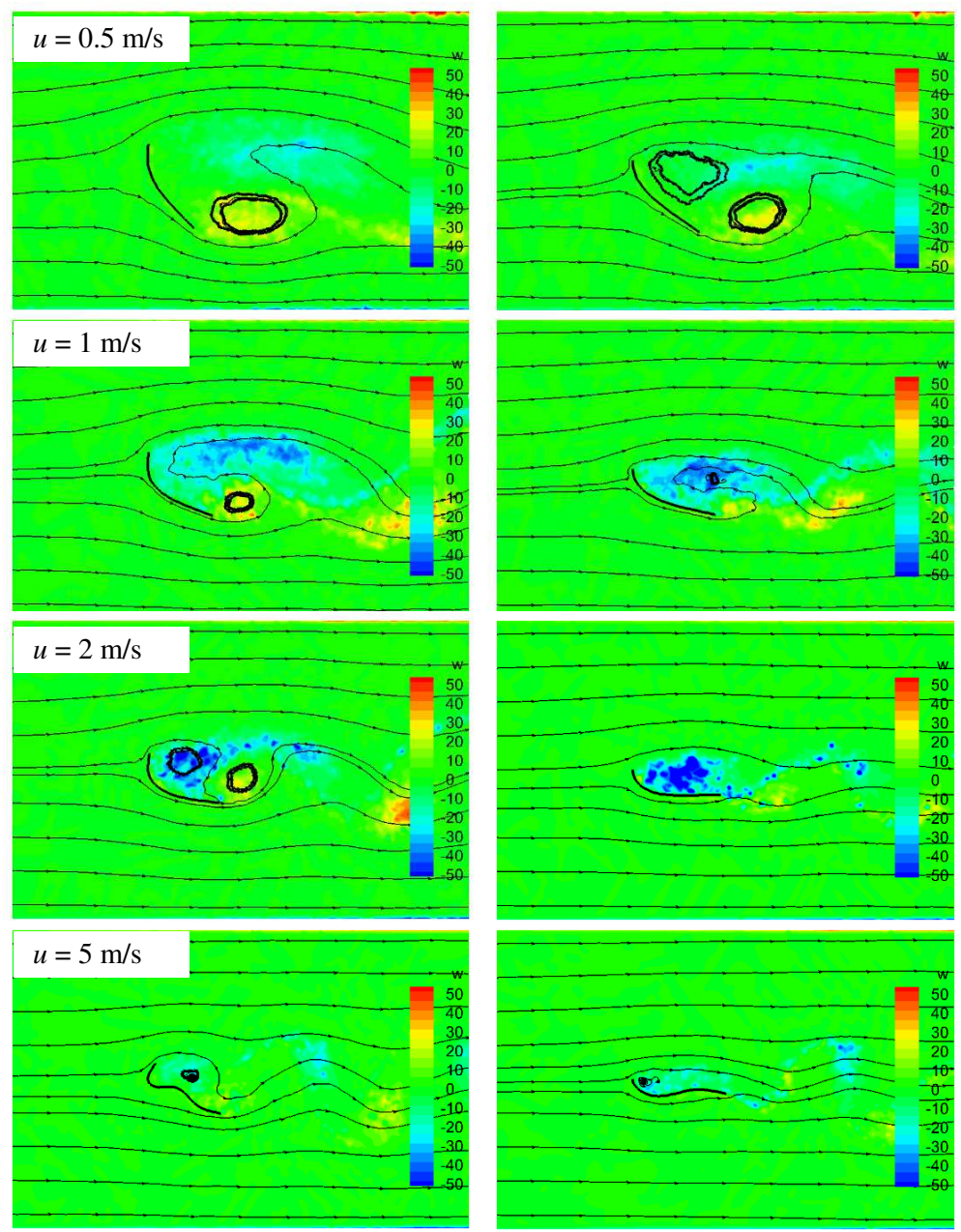

Figure 7: The streamlines for the flexible fiber of length $L=3 \mathrm{~cm}$ but different asymmetric number $e=2 / 3$ (left) and $e=1$ (right) at different flow velocities. The color shows the angular velocity of SPH particles.

\subsection{Drag force}

Fig. 8 shows the drag forces per profile length acting on the flexible fibers with the same length $L=3 \mathrm{~cm}$ but different asymmetric numbers $e=0,1 / 3,2 / 3$, and 1 . We can see from Fig. 8 that when flow velocity is less than $1.5 \mathrm{~m} / \mathrm{s}$, the drag forces on the symmetric fiber $(e=0)$ is larger than the drag forces on the asymmetric fibers $(e=1 / 3,2 / 3$ and 1$)$. It is observed that the fiber with a larger asymmetric number undergoes a less drag force. The reason is that a longer end of a fiber is easier to bend and to form streamlined shapes at low flow velocities, and a more streamlined shape led to a less drag. When the asymmetric number increases, the length of the longer end of the fiber also increases, so the drag force decreases, that is, a larger asymmetric number has a less drag. However, as the flow 


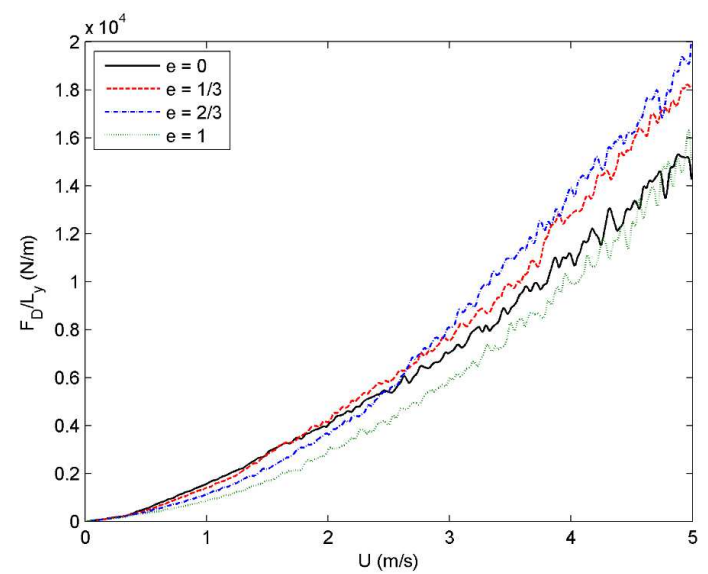

Figure 8: The fluid drag per profile length as a function of flow velocity for fibers of the same length $L=3 \mathrm{~cm}$ but different asymmetric number $e=0,1 / 3,2 / 3$ and 1 .

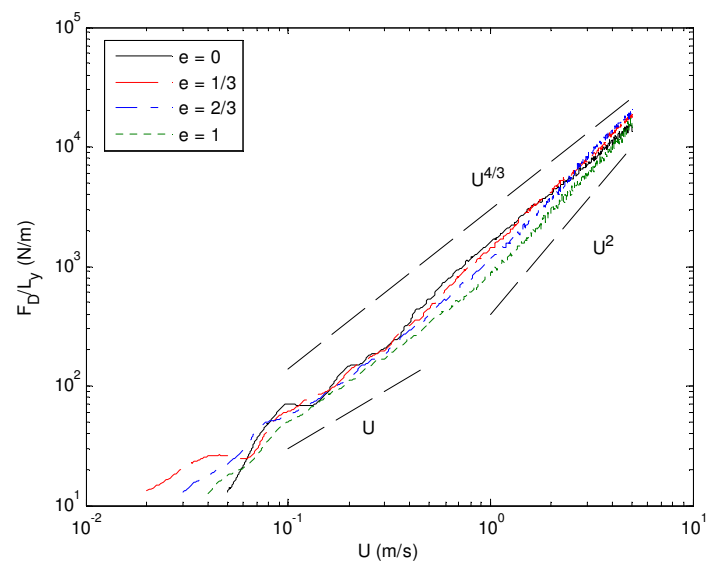

Figure 9: Log-log plots of the fluid drag per profile length as a function of flow velocity for fibers of the same length $L=3 \mathrm{~cm}$ but different asymmetric number $e=0,1 / 3,2 / 3$ and 1 .

velocity increases, the drag forces on the asymmetric fibers increase faster than the drag on the symmetric fiber. There are two possible reasons: 1) the effect of the short end of the fiber increases as flow velocity increases; 2 ) at high flow velocities, the longer end of the fiber flaps, which lead to large drag. Since the drag on an asymmetric fiber increases faster than the drag on the symmetric fiber, the former is larger than the later when the flow velocity is larger than a certain value as shown in Fig. 8, that is, the drag forces on the fibers with $e=1 / 3,2 / 3$, and 1 are larger than the drag force on the symmetric fiber with $e=0$ when flow velocity is larger than about $1.8,2.5$, and $4.9 \mathrm{~m} / \mathrm{s}$, respectively.

Fig. 9 shows the log-log plots of fluid drag for fibers with different asymmetric numbers. The data in Figs. 8 and 9 are the same except that Fig. 9 is used to show the drag 


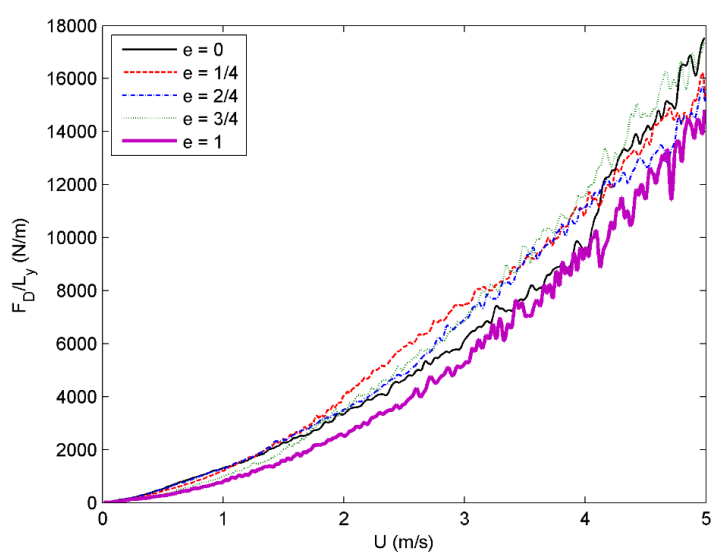

Figure 10: The fluid drag per profile length as a function of flow velocity for fibers of the same length $L=4 \mathrm{~cm}$ but different asymmetric number $e=0,1 / 4,2 / 4,3 / 4$, and 1 .

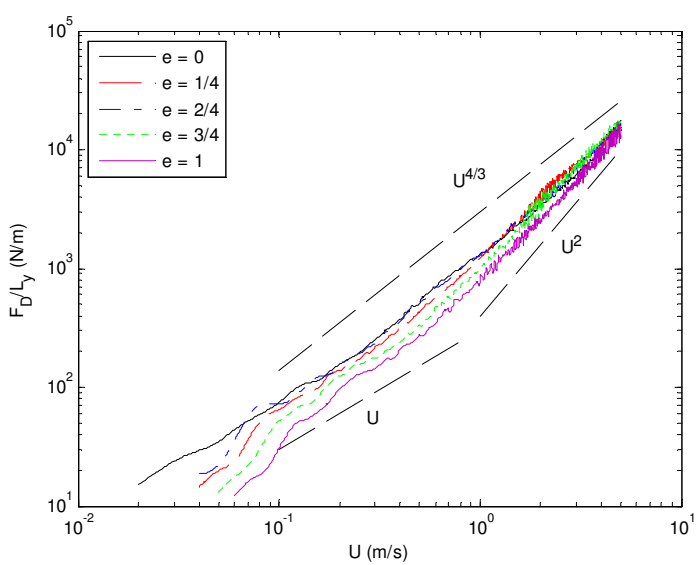

Figure 11: Log-log plots of the fluid drag per profile length as a function of flow velocity for fibers of the same length $L=4 \mathrm{~cm}$ but different asymmetric number $e=0,1 / 4,2 / 4,3 / 4$ and 1 .

scale. It is shown in Fig. 9 that at flow velocities lower than $0.4 \mathrm{~m} / \mathrm{s}$, the drag scaling of the flexible fibers is between $U$ and $U^{4 / 3}$. As flow velocity increases, the drags increase to the scale of $U^{4 / 3}$ to $U^{2}$. Fig. 9 also shows that when the flow velocity is larger than $1 \mathrm{~m} / \mathrm{s}$, the drag scale increases as the asymmetric number increases.

Figs. 10 and 11 show the drag forces per profile length for the fibers of the same length $L=4 \mathrm{~cm}$ but different asymmetric numbers. The effect of the asymmetric number on the fiber of length $L=4 \mathrm{~cm}$ is very similar to that of the fiber of length $L=3 \mathrm{~cm}$. The difference is that there is a quick increase of drag on the symmetric fiber of length $L=4 \mathrm{~cm}$ when flow velocity is larger than $4 \mathrm{~m} / \mathrm{s}$ as shown in Fig. 10. This is caused by the flapping of the fiber. 


\section{Conclusions}

In this paper, the dynamics of an asymmetric flexible fiber interacting with a viscous flow is studied numerically using the SPH-EBG coupling method. To describe the asymmetry of a flexible fiber, an asymmetric number is defined based on the lengths of the longer and shorter ends of the fiber. A series of numerical simulations with fibers of different asymmetric number from 0 to 1 were performed to study the effect of asymmetry on the fiber shape, flow field and fluid drag.

From the numerical simulations, the following conclusions can be drawn:

1. For the asymmetric fibers in viscous fluid flows, the resultant fiber shapes and flow fields are asymmetric. As flow velocity increases, Karman vortex streets are produced behind all the fibers regardless of the asymmetric numbers.

2. As the asymmetric number increases, the flexible fiber bends more on its longer free end at high flow velocities.

3. As the asymmetric number increases, the drag force decreases at low flow velocities. However, as flow velocity increases, the drag on the fiber with larger asymmetric number increases faster.

\section{Acknowledgments}

This work was supported by the National Natural Science Foundation of China (Grant Nos. 11302237 and U1530110).

\section{References}

[1] T. Y. Wu, Fish swimming and bird/insect flight, Annu. Rev. Fluid Mech., 43 (2011), 25-58.

[2] J. C. Liao, D. N. Beal, G. V. Lauder and M. S. Triantafyllou, Fish exploiting vortices decrease muscle activity, Science, 302 (2003), 1566-1569.

[3] S. Vogel, Drag and reconfiguration of broad leaves in high winds, J. Exp. Bot., 40 (1989), 941-948.

[4] S. Vogel, Drag and flexibility in sessile organisms, American Zoologist, 24 (1984), 37-44.

[5] M. J. Shelley and J. Zhang, Flapping and bending bodies interacting with fluid flows, Annu. Rev. Fluid Mech., 43 (2011), 449-465.

[6] L. Schouveiler and C. Eloy, Flow-induced draping, Phys. Rev. Lett., 111 (2013), 064301.

[7] S. Vogel, Life in Moving Fluids: the Physical Biology of Flow, Princeton University Press, Princeton, 1994.

[8] L. A. Miller, A. Santhanakrishnan, S. Jones, C. Hamlet, K. Mertens and L. Zhu, Reconfiguration and the reduction of vortex-induced vibrations in broad leaves, J. Exp. Biol., 215 (2012), 2716-2727.

[9] F. Gossellin, E. de Langre and B. A. Machado-Almeida, Drag reduction of flexible plates by reconfiguration, J. Fluid Mech., 650 (2010), 319-341. 
[10] S. Alben, M. Shelley and J. Zhang, Drag reduction through self-similar bending of a flexible body, Nature, 420 (2002), 479-481.

[11] S. Alben, M. Shelley and J. Zhang, How flexibility induces streamlining in a twodimensional flow, Phys. Fluids, 16 (2004), 1694-1713.

[12] X. F. Yang, M. B. Liu and S. L. Peng, Smoothed particle hydrodynamics and element bending group modeling of flexible fibers interacting with viscous fluids, Phys. Rev. E, 90 (2014), 063011.

[13] X. F. Yang and M. B. Liu, Bending modes and transition criteria for a flexible fiber in viscous flows, J. Hydrodyn., 28 (2016), 1043-1048.

[14] X. F. Yang, M. B. Liu, S. L. Peng and C. G. Huang, Numerical modeling of dam-break flow impacting on flexible structures using an improved SPH-EBG method, Coast. Eng., 108 (2016), 56-64.

[15] M. B. Liu and G. R. Liu, Smoothed Particle Hydrodynamics (SPH): an overview and recent developments, Arch. Comput. Methods Eng., 17 (2010), 25-76.

[16] D. Zhou and R. Wagoner, Development and application of sheet-forming simulation, J. Mater. Process Tech., 50 (1995), 1-16.

[17] S. M. Hosseini and J. J. Feng, A particle-based model for the transport of erythrocytes in capillaries, Chem. Eng. Sci., 64 (2009), 4488-4497.

[18] J. J. Monaghan, Smoothed particle hydrodynamics, Ann. Rev. Astron. Astrophys., 30 (1992), 543-574.

[19] L. B. Lucy, A numerical approach to the testing of the fission hypothesis, Astron. J., 82 (1977), 1013-1024.

[20] R. A. Gingold and J. J. Monaghan, Smoothed particle hydrodynamics: theory and application to non-spherical stars, Mon. Not. R. Astron. Soc., 181 (1977), 375-389.

[21] X. F. Yang, S. L. Peng and M. B. Liu, A new kernel function for SPH with applications to free surface flows, Appl. Math. Model., 38 (2014), 3822-3833.

[22] X. F. Yang, M. B. Liu and S. L. Peng, Smoothed particle hydrodynamics modeling of viscous liquid drop without tensile instability, Comput. Fluids, 92 (2014), 199-208.

[23] X. Yang, L. Dai and S.-C. Kong, Simulation of liquid drop impact on dry and wet surfaces using SPH method, P. Combust. Inst., 36 (2017), 2393-2399.

[24] J. P. Morris, P. J. Fox and Y. Zhu, Modeling low Reynolds number incompressible flows using SPH, J. Comput. Phys., 136 (1997), 214-226.

[25] M. B. Liu and S. M. Li, On the modeling of viscous incompressible flows with smoothed particle hydro-dynamics, J. Hydrodyn., 28 (2016), 731-745.

[26] X. F. Yang, S. L. Peng, M. B. Liu and J. R. Shao, Numerical simulation of ballast water by SPH method, Int. J. Comput. Meth., 9 (2012), 1240002.

[27] X. F. Yang and M. B. Liu, Numerical modeling of oil spill containment by boom using SPH, Sci. China Phys. Mech. Astron., 56 (2013), 315-321. 